\title{
Maximum velocity during loaded countermovement jumps obtained with an accelerometer, linear encoder and force platform: A comparison of technologies
}

\author{
Amelia Ferro $^{\mathrm{a}, *}$, Pablo Floría ${ }^{\mathrm{b}}$, Jorge Villacieros ${ }^{\mathrm{a}}$, Alejandro Muñoz-López ${ }^{\mathrm{c}}$ \\ ${ }^{a}$ Department of Sports, Faculty of Physical Activity and Sport Sciences, Universidad Politécnica de Madrid, Madrid, Spain \\ ${ }^{\mathrm{b}}$ Department of Sports and Computer, Faculty of Physical Activity and Sport Sciences, Universidad Pablo de Olavide, Sevilla, Spain \\ ${ }^{\mathrm{c}}$ Department of Human Motricity and Sports Performance, Faculty of Education Sciences, Universidad de Sevilla, Sevilla, Spain
}

\section{A R T I C L E I N F O}

\section{Article history:}

Accepted 21 July 2019

\section{Keywords:}

Jump

Kinematics

Performance

Technological tools

\begin{abstract}
A B S T R A C T
The maximum velocity (Vmax) reached during countermovement jumps (CMJ) has been considered a performance indicator to evaluate vertical jump ability. The aim of this study was to compare Vmax during loaded CMJ (CMJloaded) using three different technologies to show a criterion for selecting the more appropriate depending on its use. Nine recreationally active men performed a CMJloaded test. Five jumps were made in each of 6 series with a 20 - kg barbell $+0,+5,+10,+15,+20$ and $+25 \mathrm{~kg}$, with 2 seconds rest between the jumps and 5 minutes rest between the series to explore a wide range of speeds. Vmax was obtained from force platform, inertial device and linear encoder technologies. Bland-Altman plots and mean differences were used to compare devices. Reproducibility was tested using the intraclass correlation coefficient (ICC) for single measures and typical error (TE). All technologies showed high levels of reproducibility, ICC higher than 0.75 and TE lower than $10 \%$. There were non-significant differences in Vmax between each pair of technologies (linear encoder $2.11 \pm 0.24 \mathrm{~m} \cdot \mathrm{s}^{-1}$, accelerometer $2.11 \pm 0.26 \mathrm{~m} \cdot \mathrm{s}^{-1}$, force platform $2.12 \pm 0.24 \mathrm{~m} \cdot \mathrm{s}^{-1}$ ) reporting a very low bias. However the limits of agreement between the different technologies evaluated were high $\left( \pm 0.33 \mathrm{~m} \cdot \mathrm{s}^{-1}\right)$. In conclusion, the accelerometer, linear encoder and force platform were suitably reliable to be used to measure Vmax during loaded vertical jumps but their values were not interchangeable.
\end{abstract}

\section{Introduction}

Resistance training is considered a valuable method to improve sports performance. Coaches and strength and conditioning professionals use the vertical force application as a key factor for success in competitions such as team sports or athletics (González-Badillo and Marques, 2010). Hence, a typical exercise to improve vertical applied force during resistance training is the loaded countermovement jump (CMJloaded) (Jiménez-Reyes et al., 2016). Jump height $(\mathrm{JH})$ has been considered an indicator of the vertical force applied during the countermovement jumps (CMJ) (Samozino et al., 2010). Most devices use flight time (FT) to calculate $\mathrm{JH}$ (Buckthorpe et al., 2012; Grabski et al., 2019; Linthorne, 2001; McMahon et al., 2016; Monnet et al., 2014). Any difference

* Corresponding author at: Faculty of Physical Activity and Sport Sciences, Universidad Politécnica de Madrid, C/ Martín Fierro, 7, 28040 - Madrid, Spain.

E-mail address: amelia.ferro@upm.es (A. Ferro). between the athlete's take-off and landing position could increase the FT but not the JH. The centre of mass (COM) height in the takeoff and landing must coincide for a correct estimation of $\mathrm{JH}$ (Dias et al., 2011; Picerno et al., 2011). Recently, it has been shown that to consider the $\mathrm{COM}$ height equal to the real $\mathrm{JH}$ is a mistake (Monnet et al., 2014). Considering this technical implication, take-off velocity has been suggested as a more suitable parameter for assessing JH (Jiménez-Reyes et al., 2016). This is calculated using the impulse-momentum method from records of a force platform (García-Ramos et al., 2015; Glatthorn et al., 2011; Grabski et al., 2019; Jiménez-Reyes et al., 2016; Linthorne, 2001; Taylor et al., 2010), and this method has been shown to be more precise compared to others using force platforms (Monnet et al., 2014). Due to the difficulty of determining the precise instant of take-off, the maximum velocity (Vmax) achieved during the upward movement has been proposed as another option (GarcíaRamos et al., 2015; Jiménez-Reyes et al., 2016; Taylor et al., 2010). Although identifying the instant at which the Vmax is 
reached could be clearer than the take-off instant, few studies have used this variable to evaluate vertical jump performance. Consequently, more studies are needed exploring the utility of Vmax.

Recently, accelerometers have also been used to calculate $\mathrm{JH}$ (Casartelli et al., 2010; Magnúsdóttir et al., 2014; Picerno et al., 2011). These systems measure the acceleration related to gravity (g). Thus, when the accelerometer registers $1 \mathrm{~g}$, it can be assumed that the athlete is in flight (Casartelli et al., 2010; Magnúsdóttir et al., 2014; Picerno et al., 2011). What is more, it is possible to calculate both the time the athlete is in flight and the velocity at takeoff obtained from the single numerical integration of vertical acceleration. However, no studies have been found which use the Vmax calculated by accelerometers to evaluate vertical jump performance.

The objectives of this study were firstly to compare Vmax obtained with an accelerometer, a linear encoder and a force platform during the execution of a CMJloaded; secondly, to determine the reliability of three devices to estimate Vmax. For the first objective, it was hypothesised that there would be high agreement in the Vmax estimation among the three devices. For the second objective, that the degree of reliability to calculate the Vmax of the three devices would be similar.

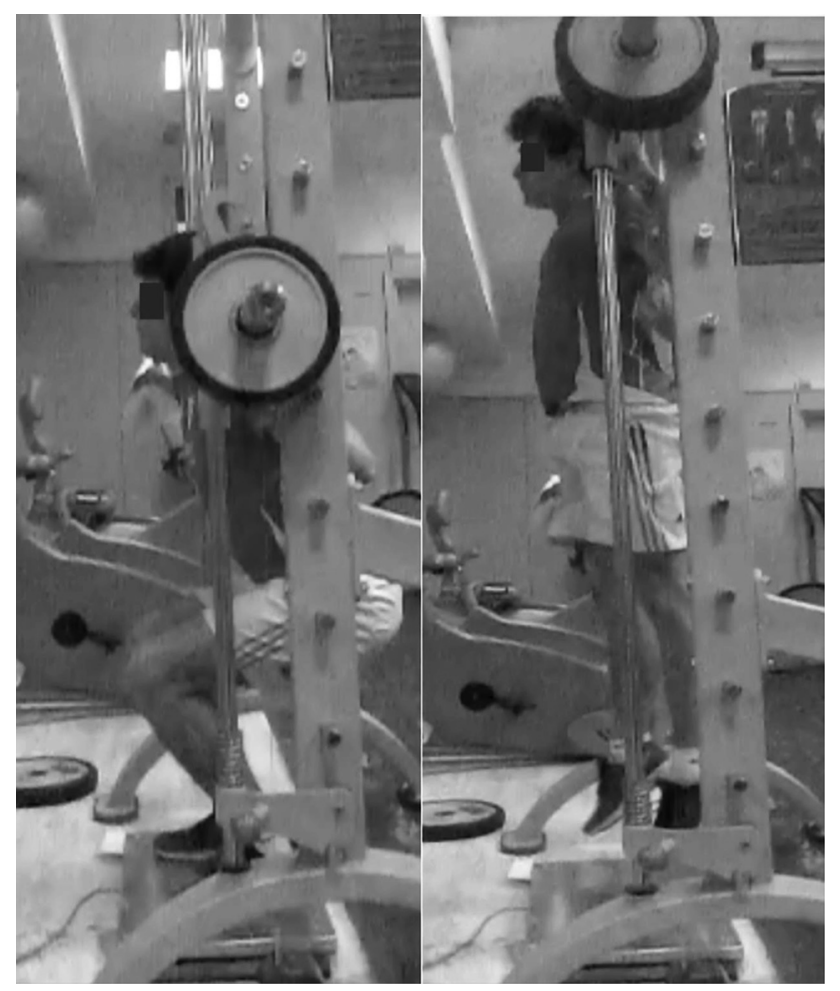

Fig. 1. A participant executing the test. The force platform was placed under the Smith-machine, the accelerometer and the cable of the encoder were fixed in the same position in the barbell.

\section{Methods}

\subsection{Subjects}

Nine recreationally active, healthy sport sciences students $(\mathrm{N}=9, \quad$ age $=20.78 \pm 2.11$ years, height $=1.76 \pm 0.05 \mathrm{~m}$, body mass $=74.50 \pm 10.55 \mathrm{~kg}$ ) were recruited for this study. None of the participants were experiencing injuries during the research task. The University-Ethics-Committee approved the study and it was undertaken according to the Helsinki Conference for research on human beings (Williams, 2008). The volunteer participants were given full information about the purpose of the study and gave their informed consent before participation.

\subsection{Experimental procedures}

The participants completed a standard 10-min warm-up composed of pedalling on a cycle ergometer, lower-body dynamic stretches, deep squats and vertical jumps. Then, each participant performed an incremental CMJloaded test on a Smith-machine (Multipower Fitness Line, Peroga, Spain) to allow only vertical displacement of the barbell. Previous studies recommended the use of the Smith-machine to determine vertical JH during the Squat-load jump (Pérez Castilla et al. 2017). Low, moderate and high loads were chosen to compare among devices ensuring a wide range of speed achieved. Five jumps were made in each of 6 series with a 20 - $\mathrm{kg}$ barbell $+0,+5,+10,+15,+20$ and $+25 \mathrm{~kg}$, with two seconds rest between jumps and $5 \mathrm{~min}$ rest between series. A force platform (Kistler Instruments Ltd., Hook, UK) was placed on the floor over a horizontal steel plate. One multisensor containing a 3Daccelerometer (Wimu, RealTrack System, Almería, Spain) and a linear encoder (SmartCoach, Stockholm, Sweden) was tightly fixed to the barbell (Fig. 1).

\subsection{Instruments}

\subsubsection{Force platform}

Data were obtained with the software BioWare V5.3.0.7 (Kistler Holding AG, Switzerland). The force platform recorded data at $1000 \mathrm{~Hz}$. To remove random noise, all measurements were lowpass-filtered at a cut-off frequency of $100 \mathrm{~Hz}$ using a 2nd-order Butterworth filter. This frequency was previously selected according to the residual analysis results (Winter, 2009). The vertical component of COM velocity was estimated using the impulse-momentum method. Net impulse was obtained by integrating the net vertical ground reaction force using the trapezoid method (Buckthorpe et al., 2012; Grabski et al., 2019; Linthorne, 2001).

\subsubsection{Accelerometer}

An inertial device (Wimu, RealTrack Systems, Almeria, Spain) which contains a 3D-accelerometer was used to measure the acceleration of the barbell at a sampling frequency of $1000 \mathrm{~Hz}$.

Table 1

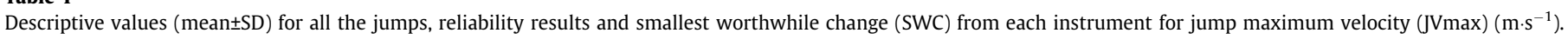

\begin{tabular}{|c|c|c|c|c|c|c|c|c|}
\hline Instrument & $\operatorname{OVmax}\left(\mathrm{m} \cdot \mathrm{s}^{-1}\right)$ & $\mathrm{JVmax} 1\left(\mathrm{~m} \cdot \mathrm{s}^{-1}\right)$ & $\mathrm{JVmax} 2\left(\mathrm{~m} \cdot \mathrm{s}^{-1}\right)$ & $\mathrm{JVmax} 3\left(\mathrm{~m} \cdot \mathrm{s}^{-1}\right)$ & ICC (95\% CI) & $\mathrm{TE}(\% \mathrm{CV})$ & $\mathrm{TE}\left(\mathrm{m} \cdot \mathrm{s}^{-1}\right)$ & $\operatorname{SWC}\left(\mathrm{m} \cdot \mathrm{s}^{-1}\right)$ \\
\hline Linear encoder & $2.11 \pm 0.24$ & $2.12 \pm 0.25$ & $2.12 \pm 0.23$ & $2.11 \pm 0.24$ & $0.944(0.913-0.966)$ & 2.87 & 0.06 & 0.12 \\
\hline Accelerometer & $2.11 \pm 0.26$ & $2.12 \pm 0.26$ & $2.11 \pm 0.24$ & $2.11 \pm 0.27$ & $0.815(0.748-0.882)$ & 4.88 & 0.09 & 0.13 \\
\hline Force platform & $2.12 \pm 0.24$ & $2.13 \pm 0.24$ & $2.11 \pm 0.24$ & $2.13 \pm 0.25$ & $0.924(0.884-0.953)$ & 2.87 & 0.06 & 0.12 \\
\hline
\end{tabular}

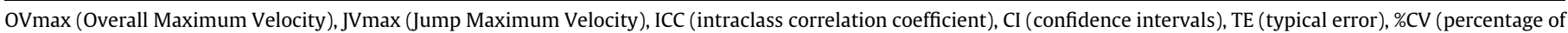
the coefficient of variation), SWC (smallest worthwhile change). 
The calibration was verified previously by checking that the accelerometer, while lying on its flat surface, constantly measured $1 \mathrm{~g}$ during $5 \mathrm{~min}$ of recording. The vertical axis was determined in relation to the axis of the earth to maintain the verticality of the movement without affecting the lateral inclination of the barbell where the device was placed. Software provided by the manufacturer (Quiko V.0.882, RealTrack System, Almería, Spain) was used to obtain the vertical velocity from acceleration data. A low-passfilter at a cut-off frequency of $10 \mathrm{~Hz}$ using a 2nd-order Butterworth filter was used to remove random noise of the data. This frequency was previously selected according to the residual analysis results (Winter, 2009).

\subsubsection{Linear encode}

The linear encoder consisted of a cable extension linear velocity transducer. Vertical instantaneous velocity was directly sampled by the device at $100 \mathrm{~Hz}$ with the software SmartCoach V4.0.12 (SmartCoach EU, Stockholm, Sweden), using a 10-point rolling average filter. Previously, the linear encoder was validated in a certified laboratory (certificate of quality ISO 90019:2008) with an average error of $0.52 \% \pm 0.17 \%$.

\subsection{Statistical analysis}

Data are shown as the mean \pm standard deviation (SD). Normal distribution was checked with the Kolmogorov-Smirnov and Shapiro-Wilk normality tests, depending on sample size. Vmax possible mean differences between pairs of instruments were calculated via a general linear model for repeated measures. In addition, Bland-Altman plot analysis was performed to compare Vmax bias between each pair of instruments and the limits of agreement (LoA). Bias, confidence intervals (CI) such as SD* 1.96 and the Bland-Altman correlation coefficient $\left(\mathrm{r}^{2}\right)$ were calculated from these plots.

To test the reproducibility of the data, the intraclass coefficient correlation (ICC) for single measures was calculated for each device and intra-trials. Additionally, the typical error (TE) as a percentage of the coefficient of variation (\%CV) and as the absolute value was calculated to provide information about the error in the measurements. In addition, the smallest worthwhile change (SWC) was calculated as $0.5 \times \mathrm{SD}$ for each data set from each instrument. Generally, an ICC higher than 0.75 and TE lower than $10 \%$ were interpreted to reflect good reliability. The level of statistical significance was set at $P<0.05$ for the mean comparisons and $P<0.01$ for the correlation analysis. Calculations were performed using SPSS Statistics 22 for Windows (IBM Co., NY. USA), and Microsoft Excel 2016 spreadsheet was made for the specific calculation of the TE.

\section{Results}

Descriptive values and reliability results for Vmax are shown in Table 1. Bland-Altman Plots are shown in Fig. 2. The linear adjustment correlation coefficient for the Bland-Altman Plots $\left(\mathrm{r}^{2}\right)$ showed stable differences within the data range for each pair of comparisons among the instruments $(r<0.009)$. The mean Bias \pm LoA was similar for all the comparison (force platform vs linear encoder $=0.02 \pm 0.33 \mathrm{~m} \cdot \mathrm{s}^{-1}$ and vs accelerometer $=0.01 \pm$ $0.33 \mathrm{~m} \cdot \mathrm{s}^{-1}$ and linear encoder vs accelerometer $=0.01 \pm$ $0.31 \mathrm{~m} \cdot \mathrm{s}^{-1}$.

The ICC for all the instruments were higher than 0.75 (linear encoder $=0.944(0.913$ to $0.966,95 \% \mathrm{CI})$, accelerometer $=0.815$ ( 0.748 to $0.88295 \% \mathrm{CI}$ ), and force platform $=0.924$ ( 0.884 to $0.95395 \% \mathrm{CI}$ ). The TE was lower than $10 \%$ for all the instruments (linear encoder $=2.87 \%$, accelerometer $=4.88 \%$, and force plat-
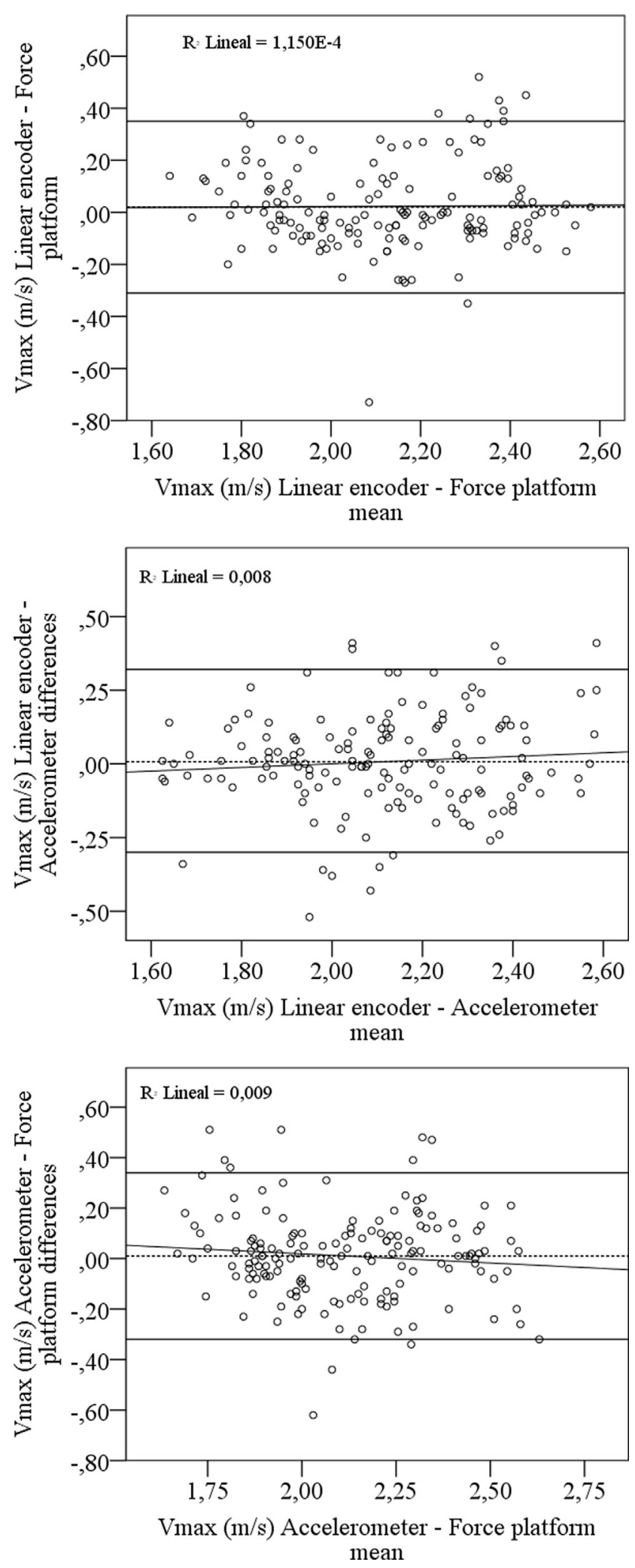

Fig. 2. Maximum velocity (Vmax) for each pair of instrument comparisons using Bland-Altman plots. The dotted horizontal line represents the absolute bias $\left(\mathrm{m} \cdot \mathrm{s}^{-1}\right)$. The upper and lower horizontal lines represent the Bland-Altman limits of agreement (LoA) $\left(\mathrm{m} \cdot \mathrm{s}^{-1}\right)$. The middle horizontal continuous line represents the Bland-Altman regression line (see methods).

form $=2.87 \%$ ). The SWC values for the linear encoder, accelerometer and force platform were $0.12 \mathrm{~m} \cdot \mathrm{s}^{-1}, 0.13 \mathrm{~m} \cdot \mathrm{s}^{-1}$ and $0.12 \mathrm{~m} \cdot \mathrm{s}^{-1}$, respectively (Table 1 ). 


\section{Discussion}

The main findings of this study confirmed our first hypothesis. No difference was found in the Vmax calculated among the three devices. In addition, the three devices were shown to be consistent when calculating Vmax with high levels of reproducibility. However, wide LoA were found in all comparison.

Previous studies have investigated the validity of an accelerometer to measure $\mathrm{JH}$, using mainly three methods: FT calculation (Casartelli et al., 2010; Castagna et al., 2013; Choukou et al., 2014; Lesinski et al., 2016; Magnúsdóttir et al., 2014; Monnet et al., 2014; Picerno et al., 2011), direct vertical acceleration measurement (Picerno et al., 2011) or velocity derived calculation (Choukou et al., 2014). Most studies reported systematic differences in the $\mathrm{JH}$ calculation between the accelerometer and other devices, such as force platforms. To our knowledge, in agreement with our results, only three studies have shown valid results on $\mathrm{JH}$ calculations compared with the force platform, although they used the FT calculation method (Castagna et al., 2013; Monnet et al., 2014; Picerno et al., 2011). Despite the low validity usually shown by other studies, they observed high reliability (Casartelli et al., 2010; Choukou et al., 2014; Lesinski et al., 2016; Picerno et al., 2011), which is in agreement with our results. The main differences found in the validity data between our study and others may be explained by the position of the accelerometer on the barbell of the Smith-machine. Recently, it has been shown that the position of the accelerometer in the COM may not necessarily reflect the athlete's real JH (Monnet et al., 2014).

The results of the present study suggest that these three devices are not interchangeable to measure $\mathrm{JH}$, due to wide LoAs found in the Vmax calculation. However, the reliability study showed that the SWC was close to $0.1 \mathrm{~m} \cdot \mathrm{s}^{-1}$ and higher than TE $\left(0.06 \mathrm{~m} \cdot \mathrm{s}^{-1}\right.$ to $\left.0.09 \mathrm{~m} \cdot \mathrm{s}^{-1}\right)$, which corresponded to a valid situation to detect changes in performance (Sánchez-Medina and González-Badillo, 2011). In addition, the reliability study showed positive values for the ICC $(>0.75)$ and TE $(<10 \%)$. For this reason, any of the three instruments were able to detect performance changes in these kinds of tests. This study shows results that will make it possible for practitioners to obtain a criterion for the selection of the instruments according to the purpose of their work, usability, price and portability.

A limitation of the present study was that the accelerometer was fixed to a barbell located in a Smith-machine ensuring a vertical path. Since it is usual in training sessions to execute load jumps without the movement restrictions, further studies are necessary to confirm these results when the jumps are executed freely for CMJloaded. Similarly, the results of this study would be limited to vertical barbell jumps. Thus, more investigation is needed to test whether the accelerometer situated on some part of the body, for example, between both scapulae, would show similar results to ours.

\section{Acknowledgements}

To the Ministry of Economy and Competitiveness of Spain for the project funding through the National Plan of Research Development Innovation (Grant ID: DEP2012-38785); to the Faculty of Physical Activity and Sport Sciences of the Universidad Politécnica de Madrid for their support and to their students for participating in the study.

\section{Conflict of interest statement}

There are no known conflicts of interest among the authors of this manuscript.

\section{References}

Buckthorpe, M., Morris, J., Folland, J.P., 2012. Validity of vertical jump measurement devices. J. Sports Sci. 30, 63-69.

Casartelli, N., Müller, R., Maffiuletti, N., 2010. Validity and reliability of the myotest accelerometric system for the assessment of vertical jump height. J. Streng. Condition. Res. 24, 3186-3193.

Castagna, C., Ganzetti, M., Ditroilo, M., Giovannelli, M., Rocchetti, A., Manzi, V., 2013. Concurrent validity of vertical jump performance assessment systems. J. Streng. Condition. Res. 27, 761-768.

Choukou, M., Laffaye, G., Taiar, R., 2014. Reliability and validity of an accelerometric system for assessing vertical jumping performance. Biol. Sport 31, 55.

Dias, J., Pupo, J., Reis, D., Borges, L., Santos, S., Moro, A., Borges, N.G., 2011. Validity of Two Methods for Estimation of Vertical Jump Height. J. Streng. Condition. Res. 25, 2034-2039.

García-Ramos, A., Stirn, I., Padial, P., Argüelles-Cienfuegos, J., Fuente, B.D.l., Strojnik, V., Feriche, B., 2015. Predicting vertical jump height from bar velocity. J. Sports Sci. Med. 14, 256

Glatthorn, J., Gouge, S., Nussbaumer, S., Stauffacher, S., Impellizzeri, F., Maffiuletti, N., 2011. Validity and reliability of optojump photoelectric cells for estimating vertical jump height. J. Streng. Condition. Res. 25, 556-560.

González-Badillo, J., Marques, M., 2010. Relationship between kinematic factors and countermovement jump height in trained track and field athletes. J. Streng. Condition. Res. 24, 3443-3447.

Grabski, J.K., Walczak, T., Michałowska, M., Pastusiak, P., Szczetyńska, M., 2019. On different methods for calculating the flight height in the vertical countermovement jump analysis. In: Arkusz, K., Będziński, R., Klekiel, T. Piszczatowski, S. (Eds.), Biomechanics in Medicine and Biology. Biomechanics 2018. Springer, Cham, pp. 242-251.

Jiménez-Reyes, P., Pareja-Blanco, F., Rodríguez-Rosell, D., Marques, M.C., GonzálezBadillo, J.J., 2016. Maximal velocity as a discriminating factor in the performance of loaded squat jumps. Int. J. Sports Phys. Perform. 11, 227.

Lesinski, M., Muehlbauer, T., Granacher, U., 2016. Concurrent validity of the Gyko inertial sensor system for the assessment of vertical jump height in female subelite youth soccer players. BMC Sports Science, Medicine and Rehabilitation, p. 8.

Linthorne, N.P., 2001. Analysis of standing vertical jumps using a force platform. Am. J. Phys. 69, 1198-1204.

Magnúsdóttir, Á., Orgilsson, B., Karlsson, B., 2014. Comparing three devices for jump height measurement in a heterogeneous group of subjects. J. Streng. Condition. Res. 28, 2837-2844.

McMahon, J.J., Jones, P.A., Comfort, P., 2016. A Correction Equation for Jump Height Measured Using the Just Jump System. Int. J. Sports Phys. Perform. 11, 555.

Monnet, T., Decatoire, A., Lacouture, P., 2014. Comparison of algorithms to determine jump height and flight time from body mounted accelerometers. Sports Eng. 17, 249-259.

Pérez-Castilla, A., McMahon, J.J., Comfort, P., García-Ramos, A., 2017. Assessment of loaded squat jump height with a free-weight barbell and Smith machine: comparison of the take-off velocity and flight time procedures. J. Streng. Cond. Res. Epub. Ahead Print. https://doi.org/10.1519/JSC.0000000000002166.

Picerno, P., Camomilla, V., Capranica, L., 2011. Countermovement jump performance assessment using a wearable 3D inertial measurement unit. J. Sports Sci. 29 139-146.

Samozino, P., Morin, J.B., Hintzy, F., Belli, A., 2010. Jumping ability: a theoretical integrative approach. J. Theor. Biol. 264 (1), 11-18.

Sánchez-Medina, L., González-Badillo, J.J., 2011. Velocity loss as an indicator of neuromuscular fatigue during resistance training. Med. Sci. Sports Exerc. 43, $1725-1734$

Taylor, K., Cronin, J., Gill, N.D., Chapman, D.W., Sheppard, J., 2010. Sources of variability in iso-inertial jump assessments. Int. J. Sports Phys. Perform. 5, 546

Williams, J.R., 2008. The Declaration of Helsinki and public health. Bull. World Health Organ. 86, 650-651.

Winter, D.A., 2009. Biomechanics and Motor Control of Human Movement. John Wiley \& Sons, INC.. 